\title{
ADAPTIVE TRACKING FOR SYSTEMS DESCRIBED BY FUNCTIONAL DIFFERENTIAL EQUATIONS
}

\author{
A. Ilchmann*, E.P. Ryan ${ }^{\dagger}$, C.J. Sangwin ${ }^{\ddagger}$ \\ * Institute of Mathematics, Technical University Ilmenau \\ Weimarer Strasse 25, 98693 Ilmenau, Germany \\ fax: $\quad+49-3677-693270$ \\ e-mail: ilchmann@mathematik.tu-ilmenau.de \\ $\dagger$ Department of Mathematical Sciences, University of Bath \\ Claverton Down, Bath BA2 7AY, UK \\ fax: +44-1225-826492 \\ e-mail: epremaths.bath.ac.uk \\ $\ddagger$ School of Mathematics \& Statistics, University of Birmingham \\ Edgbaston, Birmingham B15 2TT, UK \\ e-mail: sangwincefor.mat.bham.ac.uk
}

Keywords: adaptive control, trajectory tracking in nonlinear systems.

\begin{abstract}
An adaptive servomechanism is developed for practical tracking (with prespecified asymptotic accuracy), by the system output, of any admissible reference signal (viz. absolutely continuous and bounded with essentially bounded derivative) in the context of a class of controlled dynamical systems modelled by functional differential equations.
\end{abstract}

\section{Introduction}

Analysis and synthesis of a universal servomechanism is addressed in the context a class of controlled dynamical systems having the interconnected structure shown in Figure 1: in particular, the objective is a servomechanism which, for every system of the class, ensures practical tracking (that is, approximate tracking with prespecified asymptotic accuracy), by the system output $y$, of an arbitrary reference signal $r$ (assumed absolutely continuous and bounded with essentially bounded derivative). The dynamic block $\Sigma_{1}$, which can be influenced directly by the system input (control) $u$, is also driven by the output $w$ from the dynamic block $\Sigma_{2}$. Viewed abstractly, the block $\Sigma_{2}$ can be considered as a causal operator which maps the system output $y$ to $w$ (an internal quantity, unavailable for feedback purposes).

By way of a prototype, we remark that the adaptive servomechanism developed in this paper is applicable to the class $\mathcal{L}$ of finite-dimensional, real, linear, $M$-input $\left(u(t) \in \mathbb{R}^{M}\right), M$ output $\left(y(t) \in \mathbb{R}^{M}\right.$ ) linear systems of the form

$$
\dot{x}(t)=A x(t)+B u(t), \quad y(t)=C x(t), \quad x(0)=x^{0} \in \mathbb{R}^{N}
$$

with the minimum-phase property

$$
\operatorname{det}\left[\begin{array}{cc}
s I-A & B \\
C & 0
\end{array}\right] \neq 0 \quad \forall s \in\{z \in \mathbb{C} \mid \operatorname{Re} z \geq 0\}
$$

and having high-frequency gain $C B \in \mathbb{R}^{M \times M}$ with spectrum in the open right half complex plane.

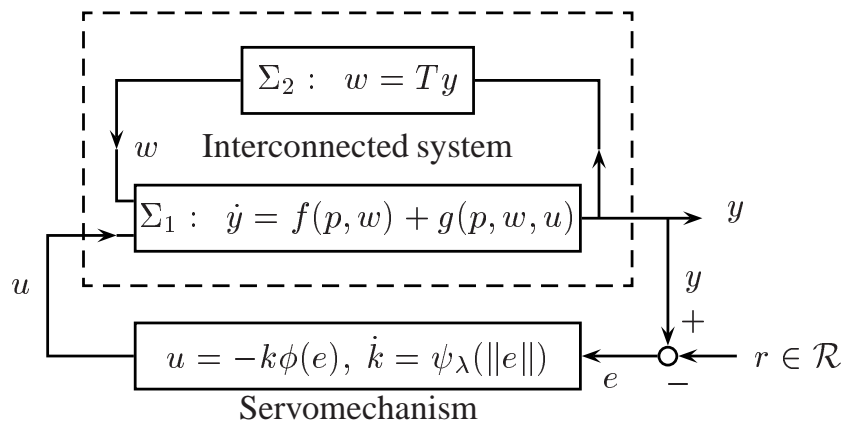

Figure 1: Interconnected subsystems $\Sigma_{1}, \Sigma_{2}$ and servomechanism

It was shown by Willems \& Byrnes [15] (see, also, the seminal work by Morse [10], [8] and [9]) that every member of the latter linear class $\mathcal{L}$ is stabilized by the following adaptive high-gain output feedback strategy

$$
u(t)=-k(t) y(t), \quad \dot{k}(t)=\|y(t)\|^{2}, \quad k(0)=k^{0} .
$$

Special features of this strategy are its simplicity and the absence of any plant identification mechanism. The strategy has been extended, by the introduction of a dead-zone in the gain adaptation (see, [5]), to achieve practical tracking (that is, tracking with prescribed asymptotic accuracy quantified by $\lambda>0)$. Specifically, the strategy

$$
u(t)=-k(t) e(t), \quad \dot{k}(t)=d_{\lambda}^{2}(\|e(t)\|), \quad k(0)=k^{0},
$$

where, for $\lambda>0, d_{\lambda}$ denotes the distance function given by $d_{\lambda}(\xi):=\max \{|\xi|-\lambda, 0\}$ and $e(t):=y(t)-r(t)$ denotes the tracking error between output and reference signal $r$, the latter 
being any bounded absolutely continuous function with essentially bounded derivative. A particular feature of the strategy (3) is that no internal model for the reference signal dynamics is required. Generalizations to various classes of nonlinear systems are contained in $[1,3,4,5,11,12]$.

The novelty of the present paper is that we enlarge considerably the underlying class of systems (compared to those considered in the above cited works). The enlarged class encompasses, for example, interconnections with systems which exhibit the input-to-state stability (ISS) property [14], systems with delays (both point and distributed) and systems with hysteretic nonlinearities. Moreover, the simplicity of the adaptation mechanism is preserved.

Notation: For $I \subset \mathbb{R}$ an interval, $C\left(I ; \mathbb{R}^{N}\right)$ denotes the set of continuous functions $I \rightarrow \mathbb{R}^{N}$. For $x: I \rightarrow \mathbb{R}^{N}$, the restriction of $x$ to $J \subset I$ is denoted by $\left.x\right|_{J} . L_{\text {loc }}^{\infty}\left(I ; \mathbb{R}^{N}\right)$ denotes the space of measurable locally essentially bounded functions $x$ : $I \rightarrow \mathbb{R}^{N}$. As before, for $\lambda>0, d_{\lambda}$ denotes the Euclidean distance function for $[-\lambda, \lambda]$ given by $d_{\lambda}(\xi):=\max \{|\xi|-\lambda, 0\}$, $\mathcal{R}$ denotes the space of bounded absolutely continuous functions $\mathbb{R} \rightarrow \mathbb{R}^{M}$ with essentially bounded derivative (which, appropriately normed, can be identified as the Sobolev space $\left.W^{1, \infty}\left(\mathbb{R} ; \mathbb{R}^{M}\right)\right)$. We write $\mathbb{R}_{+}:=[0, \infty) . \mathcal{K}$ denotes the class of continuous, strictly increasing functions $\alpha: \mathbb{R}_{+} \rightarrow \mathbb{R}_{+}$ with $\alpha(0)=0 . \mathcal{K} \mathcal{L}$ is the class of functions $\gamma: \mathbb{R}_{+}^{2} \rightarrow \mathbb{R}_{+}$ such that for each $t \in \mathbb{R}_{+}, \gamma(\cdot, t)$ is of class $\mathcal{K}$ and for each $s \in \mathbb{R}_{+}, \gamma(s, \cdot)$ is decreasing with $\gamma(s, t) \rightarrow 0$ as $t \rightarrow \infty$. Finally, a subclass $\mathcal{J}$ of $\mathcal{K}$ is introduced:

$$
\begin{aligned}
\mathcal{J}:=\left\{\alpha \in \mathcal{K} \mid \forall \delta \in \mathbb{R}_{+} \exists \Delta\right. & \in \mathbb{R}_{+}: \\
& \alpha(\delta \tau) \leq \Delta \alpha(\tau) \forall \tau \geq 0\} .
\end{aligned}
$$

\section{The class of systems}

We extend the above-mentioned prototypical results to a class of infinite-dimensional, nonlinear, $M$-input $\left(u(t) \in \mathbb{R}^{M}\right), M$ output $\left(y(t) \in \mathbb{R}^{M}\right)$ systems $(p, f, g, T)$, having the same structure as in Figure 1, and modelled by a controlled nonlinear functional differential equation of the form

$$
\left.\begin{array}{l}
\dot{y}(t)=f(p(t),(T y)(t))+g(p(t),(T y)(t), u(t)), \\
\left.y\right|_{[-h, 0]}=y^{0} \in C\left([-h, 0] ; \mathbb{R}^{M}\right)
\end{array}\right\}
$$

where $h \geq 0$ quantifies the "memory" of the system ( $h=0$ in the linear prototype), $p(\cdot)$ may be thought of as a (bounded) disturbance term and $T$ is a nonlinear causal operator.

Definition 1 (System class $\mathcal{S}$ ) Let $h \geq 0$ and $\alpha_{f}, \alpha_{T} \in \mathcal{J}$. $\mathcal{S}=\mathcal{S}\left(h, \alpha_{f}, \alpha_{T}\right)$ denotes the class of $M$-input, $M$-output systems of the form (4) with the properties (wherein $P, Q \in \mathbb{N}$ are arbitrary):

1. $p \in L^{\infty}\left([-h, \infty) ; \mathbb{R}^{P}\right)$;

2. $f: \mathbb{R}^{P} \times \mathbb{R}^{Q} \rightarrow \mathbb{R}^{M}, Q \in \mathbb{N}$, is continuous and, for every compact set $C \subset \mathbb{R}^{P}$, there exists $c_{f} \geq 0$ such that $\|f(p, w)\| \leq c_{f}\left[1+\alpha_{f}(\|w\|)\right]$ for all $(p, w) \in \bar{C} \times \mathbb{R}^{Q} ;$
3. $g: \mathbb{R}^{P} \times \mathbb{R}^{Q} \times \mathbb{R}^{M} \rightarrow \mathbb{R}^{M}$ is continuous and, for every compact set $C \subset \mathbb{R}^{P}$, there exists positive definite, symmetric $G \in \mathbb{R}^{M \times M}$ such that

$$
\langle G u, g(p, w, u)\rangle \geq\|u\|^{2}
$$

for all $(p, w, u) \in C \times \mathbb{R}^{Q} \times \mathbb{R}^{M}$;

4. $T: C\left([-h, \infty) ; \mathbb{R}^{M}\right) \rightarrow L_{\mathrm{loc}}^{\infty}\left(\mathbb{R}_{+} ; \mathbb{R}^{Q}\right)$ is a causal operator with the property that there exist $\alpha_{T} \in \mathcal{J}$ and constant $c_{T} \geq 0$ such that, for all $y \in C\left([-h, \infty) ; \mathbb{R}^{N}\right)$,

$\|(T y)(t)\| \leq c_{T}\left[1+\max _{s \in[0, t]} \alpha_{T}(\|y(s)\|)\right] \quad$ for all $t \in \mathbb{R}_{+}$.

For convenience, we denote a system of class $\mathcal{S}\left(h, \alpha_{f}, \alpha_{T}\right)$ by $(p, f, g, T) \in \mathcal{S}\left(h, \alpha_{f}, \alpha_{T}\right)$ and, whenever $h$ and the functions $\alpha_{f}$ and $\alpha_{T}$ are contextually evident, we simply write $\mathcal{S}$ in place of $\mathcal{S}\left(h, \alpha_{f}, \alpha_{T}\right)$.

\section{The control objective}

Given $h \geq 0$ and $\alpha_{f}, \alpha_{T} \in \mathcal{J}$, the control objective is to determine continuous functions

$$
\phi: \mathbb{R}^{M} \rightarrow \mathbb{R}^{M}, \quad \psi_{\lambda}: \mathbb{R}_{+} \rightarrow \mathbb{R}_{+}
$$

(the latter parameterized by $\lambda>0$ ) such that, for each system $(p, f, g, T) \in \mathcal{S}\left(h, \alpha_{f}, \alpha_{T}\right)$ and every reference signal $r \in \mathcal{R}$ (see Notation above), the control

$$
\left.\begin{array}{l}
u(t)=-k(t) \phi(y(t)-r(t)) \\
\dot{k}(t)=\psi_{\lambda}(\|y(t)-r(t)\|) \\
\left.k\right|_{[-h, 0]}=k^{0}
\end{array}\right\}
$$

(where $k^{0}$ may be assumed to be a constant non-negative-realvalued function) applied to (4) yields a closed-loop system with the following properties:

(i) the initial-value problem $(4,5)$ has a solution and every solution has a maximal extension $(y, k):[-h, \omega) \rightarrow$ $\mathbb{R}^{M} \times \mathbb{R}$ (continuous, with $\left.(y, k)\right|_{[-h, 0]}=\left(y^{0}, k^{0}\right)$, such that $\left.(y, k)\right|_{[0, \omega)}$ is absolutely continuous and satisfies the differential equations in (4), (5) for almost all $t$, and $(y, k)$ has no right extension which is also a solution);

(ii) every maximally-extended solution is bounded (and so $\omega=\infty)$ and, by monotonicity of $k, \lim _{t \rightarrow \infty} k(t)$ exists and is finite;

(iii) the tracking error $e(t):=y(t)-r(t)$ approaches the closed ball of radius $\lambda>0$ centred at $0 \in \mathbb{R}^{M}$; equivalently, $d_{\lambda}(\|e(t)\|) \rightarrow 0$ as $t \rightarrow \infty$.

\section{The main result}

We preface the main result with the remark that the defining Properties 1-4 of the system class $\mathcal{S}$ may not, in general, constitute sufficient conditions to guarantee existence of a solution of 
the initial-value problem $(4,5)$. However, as detailed in $[13,6]$, if additional regularity is imposed on the operator $T$, then existence of a solution is assured. This additional regularity corresponds to satisfaction of [6, Defn 1: Property 2(b)] (see also [13, Defn 4: Property 2(b)]): the essence of this extra condition (which, for reasons of brevity, we do not elucidate here) is the imposition of a rather weak "local Lipschitz" property on $T$ : suffice it to remark here that the operator $T$ in each example in Section 5 below exhibits the requisite property. In the present paper, we will refer loosely to the property as the "weak local Lipschitz property", abbreviated as the "wll-property".

Theorem 2 Let $h \geq 0, \alpha_{f}, \alpha_{T} \in \mathcal{J}, \lambda>0$ and

$$
\alpha: \xi \mapsto\left[\xi+\alpha_{f} \circ \alpha_{T}(\xi)\right] / 2
$$

Define the continuous functions $\phi: \mathbb{R}^{M} \rightarrow \mathbb{R}^{M}$ and $\psi_{\lambda}:$ $\mathbb{R}_{+} \rightarrow \mathbb{R}_{+}$by:

$$
\begin{aligned}
& \phi(0):=0, \quad \phi(e):=\alpha(\|e\|)\|e\|^{-1} e, e \neq 0 \\
& \psi_{\lambda}(0):=0, \quad \psi_{\lambda}(\xi):=\max \{0,[\alpha(\xi)-\alpha(\lambda)]\} / \xi, \quad \xi>0 .
\end{aligned}
$$

Let $r \in \mathcal{R},(p, f, g, T) \in \mathcal{S}\left(h, \alpha_{f}, \alpha_{T}\right)$ and assume that $T$ has the wll-property. Then, for each $\left(y^{0}, k^{0}\right) \in C\left([-h, 0] ; \mathbb{R}^{M+1}\right)$, the feedback strategy (5) applied to (4) yields a closed-loop system which achieves the control objectives (i)-(iii).

Remarks. A sketch of the proof of Theorem 2 is provided below (full details can be found in [6]). We remark that, for illustrative purposes, in the above theorem we have given simple choices for the controller functions $\phi$ and $\psi_{\lambda}$. In fact, there is a plurality of possibilities in those choices [6]: practical considerations might suggest appropriate alternatives.

\section{Sketch proof.}

(a) For each $\left(y^{0}, k^{0}\right) \in C\left([-h, 0] ; \mathbb{R}^{M+1}\right)$, [6, Theorem 3] (see also [13, Theorem 1]) assures existence of a solution of the initial-value problem (4)-(5): moreover, every solution can be extended to a maximal solution $(y, k):[-h, \omega) \rightarrow \mathbb{R}^{M+1}$.

(b) Let $(y, k)$ be a maximal solution. Writing $e(t)=y(t)-r(t)$ and $V(t):=\frac{1}{2}\langle e(t), G e(t)\rangle$, it can be shown that, for some constant $c_{1}>0$,

$$
\begin{aligned}
\dot{V}(t) \leq c_{1}[1 & \left.+\max _{s \in[0, t]} \alpha_{f}\left(\alpha_{T}(\|e(s)\|)\right)\right]\|e(t)\| \\
& -k(t) \alpha(\|e(t)\|)\|e(t)\| \text { a.a. } t \in[0, \omega) .
\end{aligned}
$$

(c) Seeking contradiction, suppose $e$ is unbounded. For each $n \in \mathbb{N}$, define

$$
\begin{aligned}
& \tau_{n}:=\inf \left\{t \in[0, \omega) \mid \quad c_{2} \sqrt{V(t)}=n+1+\gamma\right\} \\
& \sigma_{n}:=\sup \left\{t \in\left[0, \tau_{n}\right] \mid \quad c_{2} \sqrt{V(t)}=n+\gamma\right\}
\end{aligned}
$$

where $c_{2}:=\sqrt{2 /\|G\|}$ and $\gamma:=\|e(0)\|+1$. A (lengthy) calculation now yields, for some constants $c_{3}, c_{4}>0$,

$$
\begin{aligned}
\dot{V}(t) \leq\left[c_{3}-k(t)\right] \alpha(\|e(t)\|)\|e(t)\| & \\
& \leq c_{4} \alpha\left(c_{2} \sqrt{V(t)}\right) \sqrt{V(t)} \quad \forall t \in\left[\sigma_{n}, \tau_{n}\right]
\end{aligned}
$$

whence, for some $c_{5}>0$,

$$
\begin{aligned}
2 \ln \left(\frac{n+1+\gamma}{1+\gamma}\right) & \leq c_{5} \sum_{j=1}^{n} \int_{\sigma_{j}}^{\tau_{j}} \psi_{\lambda}(\|e(t)\|) d t \\
& \leq c_{5} k\left(\tau_{n}\right) \quad \forall n \in \mathbb{N} .
\end{aligned}
$$

Therefore, $k(\cdot)$ is unbounded and so, by (7), for $n^{*}$ sufficiently large,

$$
\dot{V}(t) \leq-c_{3} \alpha(\|e(t)\|)\|e(t)\|<0 \quad \text { for a.a. } t \in\left[\sigma_{n^{*}}, \tau_{n^{*}}\right],
$$

which contradicts the fact that $V\left(\tau_{n^{*}}\right)>V\left(\sigma_{n^{*}}\right)$. Therefore, $e:[-h, \omega) \rightarrow \mathbb{R}^{M}$ is bounded.

(d) By continuity of $\psi_{\lambda}$ and boundedness of $e$, it follows that $\dot{k}$ is bounded and so $k$ is bounded on every compact subinterval $[0, T] \subset[0, \omega)$. Therefore $\omega=\infty$.

(e) Next, we prove boundedness of $k$. By boundedness of $e$ and (6), there exists a constant $c_{6}>0$ such that

$$
\dot{V}(t) \leq c_{6}-k(t) \beta(V(t)) \quad \text { a.a. } t \in[0, \infty),
$$

where $\beta \in \mathcal{K}$ is given by $\beta(s)=\alpha\left(c_{2} \sqrt{s}\right) c_{2} \sqrt{s}$. Seeking a contradiction, suppose that $k$ is unbounded. Then, by (8), there exists $\tau \in[0, \infty)$ such that $\|e(t)\|<\lambda$ for all $t \in[\tau, \infty)$ and so $\dot{k}(t)=0$ for all $t \in[\tau, \infty)$, which contradicts the supposition of unboundedness of $k$. Therefore, the monotone function $k$ is bounded and so $\lim _{t \rightarrow \infty} k(t)$ exists and is finite. We have now established control objectives (i) and (ii). It remains to establish objective (iii).

(f) By boundedness of $e, k$ and essential boundedness of $\dot{r}$, we may conclude from (4)-(5) that $e$ has essentially bounded derivative and so $e$ is uniformly continuous. By boundedness and uniform continuity of $e$, together with continuity of $\psi_{\lambda}$, it follows that $\psi_{\lambda}(\|e(\cdot)\|)$ is uniformly continuous. By boundedness of $k$,

$$
\int_{0}^{\infty} \psi_{\lambda}(\|e(t)\|) d t<\infty .
$$

By Barbălat's Lemma [2], we conclude that $\psi_{\lambda}(\|e(t)\|) \rightarrow 0$ as $t \rightarrow \infty$ whence, recalling that $\psi_{\lambda}^{-1}(0)=[0, \lambda]$, objective (iii).

\section{Examples}

\subsection{The prototype class of finite-dimensional linear sys- tems}

Consider again the prototype class $\mathcal{L}$ of finite-dimensional, linear, minimum-phase systems (1) of Section 1, where "highfrequency" gain $C B$ has spectrum in the open right half plane. Under a suitable coordinate transformation (see, for example, in [3, Proposition 2.1.2]), every system in $\mathcal{L}$ can be expressed in the form of two coupled subsystems

$$
\left.\begin{array}{ll}
\dot{y}(t)=A_{1} y(t)+A_{2} z(t)+C B u(t), & y(0)=y^{0} \\
\dot{z}(t)=A_{3} y(t)+A_{4} z(t), & z(0)=z^{0}
\end{array}\right\}
$$


with $y(t), u(t) \in \mathbb{R}^{M}, z(t) \in \mathbb{R}^{N-M}$, and where (by the minimum-phase property) $A_{4}$ has spectrum in the open left half complex plane. Introducing the linear operator $T$ given by

$$
(T y)(t):=A_{1} y(t)+A_{2} \int_{0}^{t} \exp \left(A_{4}(t-s)\right) A_{3} y(s) d s
$$

and the function $p$ given by $p(t):=A_{2} \exp \left(A_{4} t\right) z^{0}$, then, with respect to our operator theoretic viewpoint, system (9) can be interpreted as (4) with $f$ and $g$ given by

$$
f:(p, w) \mapsto p+w, \quad g:(p, w, u) \mapsto C B u .
$$

Since the eigenvalues of $A_{4}$ have strictly negative real part, $p(\cdot)$ is evidently bounded (whence Property 1 of Definition 1) and, by (10), Property 4 holds with $h=0, \alpha_{T}=$ id (the identity map) and, moreover, $T$ has the wll-property. The function $f$ clearly satisfies Property 2 with $\alpha_{f}=$ id. Since the eigenvalues of $C B$ have strictly positive real part, there exists $G=G^{T}$ > 0 such that $G C B+(C B)^{T} G>0$ and so Property 3 holds. Therefore, system $(p, f, g, T)$ is of class $\mathcal{S}(0, \mathrm{id}, \mathrm{id})$ and, by Theorem 2, the control

$$
\left.\begin{array}{l}
u(t)=-k(t) e(t) \\
\dot{k}(t)=d_{\lambda}(\|e(t)\|)\|e(t)\|^{-1} \\
k(0)=k^{0} \geq 0
\end{array}\right\}
$$

(wherein the righthand side of the second equation is interpreted as zero for $e(t)=0$ ) achieves the control objectives (i)-(iii).

\subsection{Infinite-dimensional linear systems}

The above results on finite-dimensional linear systems extend directly to infinite-dimensional systems of the form (9), where now $\left(A_{4}, A_{3}, A_{2}\right)$ are assumed to be the generating operators of a regular (in the sense of Weiss [16]) linear system with state space $X$ (a Hilbert space): $A_{4}$ is the generator of a strongly continuous semigroup $\left(S_{t}\right)_{t>0}$ of bounded linear operators on $X ; A_{3}$ is an admissible input operator; $A_{2}$ is an observation operator, assumed bounded. For brevity, we omit details here but remark that our formulation encompasses single-input, singleoutput systems of coupled ordinary and partial linear differential equations of the form

$$
\begin{aligned}
& \dot{y}(t)=p(t)+a_{0} y(t)+\frac{c_{1}}{2 \epsilon} \int_{\xi_{o}-\epsilon}^{\xi_{o}+\epsilon} v(t, \xi) d \xi+b u(t) \\
& v_{t t}(t, \xi)=a_{1} v_{\xi \xi}(t, \xi)-a_{2} v_{t}(t, \xi)+c_{0} \delta\left(\xi-\xi_{i}\right) y(t) \\
& v(t, 0)=0=v(t, 1)
\end{aligned}
$$

with arbitrary parameters $a_{0}, a_{1}, a_{2}, c_{0} \in \mathbb{R}$ and $\xi_{o}, \xi_{i} \in(0,1)$ and $\epsilon>0$. For such systems, the control (11) is such that Theorem 2 is applicable and so the control objectives (i)-(iii) are achieved.

\subsection{Systems with delays and hysteresis}

Consider the general system (4) and assume that $p, f, g$ are such that Properties 1-3 of Definition 1 hold. With regard to the casual operator $T$ (equivalently, subsystem $\Sigma_{2}$ of Figure 1), our formulation admits delay and hysteresis operators. For example, with $p, q_{1}, q_{2}, q_{3} \in L^{\infty}\left(\mathbb{R}_{+} ; \mathbb{R}\right)$, an admissible system with point and distributed delays is given by

$$
\begin{aligned}
\dot{y}(t)=p(t) y(t)+q_{1}(t) & y\left(t-h_{1}\right)+q_{2}(t) y^{2}\left(t-h_{2}\right) \\
& +\int_{-h_{3}}^{0} q_{3}(s) y^{3}(t+s) d s+b u(t) .
\end{aligned}
$$

As a second example, we remark that a general class of nonlinear operators $C\left(\mathbb{R}_{+} ; \mathbb{R}\right) \rightarrow C\left(\mathbb{R}_{+} ; \mathbb{R}\right)$, which includes many physically motivated hysteretic effects, is defined via assumptions (N1)-(N8) of [7, Section 3]. Such nonlinear operators are admissible within the current framework. Examples of such operators, including relay hysteresis, backlash hysteresis, elastic-plastic hysteresis and Preisach operators, are detailed in [7, Section 5]. By way of illustration, operators $T$, corresponding to relay and backlash hysteresis as depicted in Figures 2 and 3 , are admissible within our formulation. The reader is referred to [13] for further details.

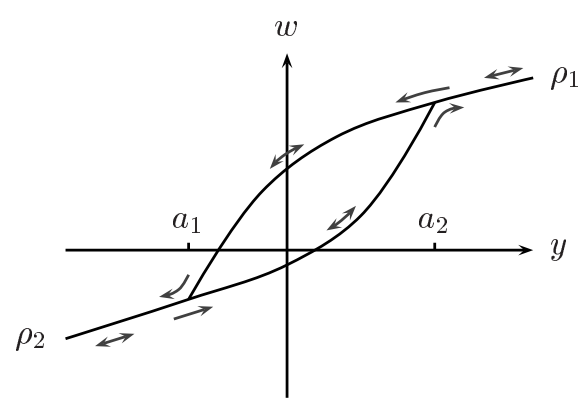

Figure 2: Relay-type hysteresis

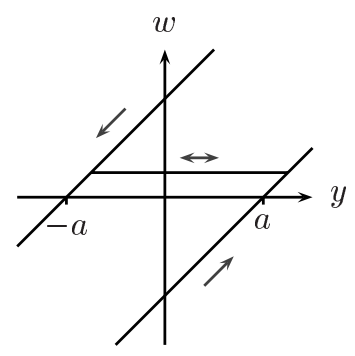

Figure 3: Backlash hysteresis

\subsection{Input-to-state stable systems}

Another class of admissible operators $T$, representing subsystem $\Sigma_{2}$ of Figure 1, is the class of input-to-state (ISS) systems. Let $Z: \mathbb{R}^{L} \times \mathbb{R}^{M} \rightarrow \mathbb{R}^{L}$ be locally Lipschitz with $Z(0,0)=0$. For $y \in L_{\text {loc }}^{\infty}\left(\mathbb{R}_{+} ; \mathbb{R}^{M}\right)$, let $z\left(\cdot, z^{0}, y\right)$ denote the unique maximal solution of the initial-value problem

$$
\dot{z}(t)=Z(z(t), y(t)), \quad z(0)=z^{0} \in \mathbb{R}^{L} .
$$


Assume that the system is input-to-state stable (ISS) [14], that is, there exist functions $\theta \in \mathcal{K} \mathcal{L}$ and $\gamma \in \mathcal{K}$ such that, for all $\left(z^{0}, y\right) \in \mathbb{R}^{L} \times L_{\mathrm{loc}}^{\infty}\left(\mathbb{R}_{+} ; \mathbb{R}^{M}\right)$,

$$
\begin{array}{r}
\left\|z\left(t, z^{0}, y\right)\right\| \leq \theta\left(\left\|z^{0}\right\|, t\right)+\text { ess-sup }_{s \in[0, t]} \gamma(\|y(s)\|) \\
\text { for all } t \geq 0 .
\end{array}
$$

Let $W: \mathbb{R}^{L} \rightarrow \mathbb{R}^{Q}$ be locally Lipschitz and such that there exists $c>0$ such that $\|W(z)\| \leq c\|z\|$ for all $z \in \mathbb{R}^{L}$. Now consider system (12) with output $w$ given by

$$
w(t)=W\left(z\left(t, z^{0}, y\right)\right)
$$

Fix $z^{0} \in \mathbb{R}^{L}$ arbitrarily. Define the operator

$$
T: C\left(\mathbb{R}_{+} ; \mathbb{R}^{M}\right) \rightarrow L_{\mathrm{loc}}^{\infty}\left(\mathbb{R}_{+} ; \mathbb{R}^{Q}\right)
$$

by

$$
(T y)(t):=W\left(z\left(t, z^{0}, y\right)\right), \quad t \geq 0
$$

It can readily be verified that this operator is admissible within our formulation. The reader is referred to $[13,6]$ for further details and generalizations of this operator class.

\section{References}

[1] F. Allgöwer, J. Ashman, A. Ilchmann. "High-gain adaptive $\lambda$-tracking for nonlinear systems", Automatica, 33, pp. 881-888, (1997).

[2] I. Barbălat. "Systèmes d'équations différentielles d'oscillations non linéaires", Revue de Mathématiques Pures et Appliquées, Bucharest, IV, pp. 267-270, (1959).

[3] A. Ilchmann. Non-identifier-based high-gain adaptive control, Springer-Verlag, London, (1993).

[4] A. Ilchmann. "Adaptive $\lambda$-tracking for polynomial minimum phase systems", Dynamics and Stability of Systems, 13, pp. 341-371, (1998).

[5] A. Ilchmann, E.P. Ryan. "Universal $\lambda$-tracking for nonlinearly perturbed systems in the presence of noise", Automatica, 30, pp. 337-346, (1994).

[6] A. Ilchmann, E.P. Ryan, C.J. Sangwin. "Adaptive tracking control for systems modelled by functional differential equations", preprint, (2000).

[7] H. Logemann, A.D. Mawby. "Low-gain integral control of infinite dimensional regular linear systems subject to input hysteresis", Advances in Mathematical Systems Theory, Eds.: F. Colonius, U. Helmke, D. Prätzel-Wolters, F. Wirth, Birkhäuser Verlag, Boston, pp. 255-293, (2000).

[8] I.M.Y. Mareels. "A simple selftuning controller for stably invertible systems", Systems and Control Letters, 4, pp. 516, (1984).
[9] B. Mårtensson. "The order of any stabilizing regulator is sufficient a priori information for adaptive stabilization", Systems and Control Letters, 6, pp. 87-91, (1985).

[10] A.S. Morse. "Recent problems in parameter adaptive control", Outils et Modèles Mathématiques pour l'Automatique, l'Analyse de Systèmes et le Traitment du Signal (I.D. Landau, Ed.), (Editions du CNRS 3, Paris), pp. 733-740, (1983).

[11] E.P. Ryan. "A nonlinear universal servomechanism", IEEE Trans. Auto. Control, 39, pp. 753-761, (1994).

[12] E.P. Ryan. "An integral invariance principle for differential inclusions with applications in adaptive control", SIAM J. Control \& Opt., 36, pp. 960-980, (1998).

[13] E.P. Ryan, C.J. Sangwin. "Functional differential inclusions and adaptive stabilization", Int. J. Control, 74, pp. 77-90, (2001).

[14] E.D. Sontag. "Smooth stabilization implies coprime factorization", IEEE Transactions on Automatic Control, 34, pp. 435-443, (1989).

[15] J.C. Willems, C.I. Byrnes. "Global adaptive stabilization in the absence of information on the sign of the high frequency gain", Lect. Notes in Control and Inf. Sciences, Springer-Verlag, Berlin, 62, pp. 49-57, (1984).

[16] G. Weiss. "Transfer-functions of regular linear-systems, part 1: Characterization of regularity", Transactions of the American Mathematical Society, 342, pp. 827-854, (1994). 